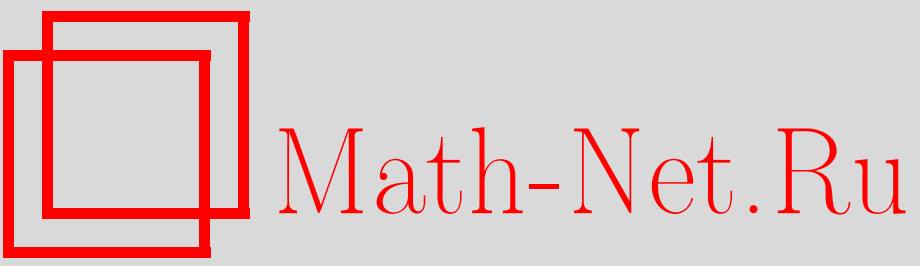

П. А. Кларксон, Э. Л. Менсфилд, Х. Н. Вебстер, О соотношении между дискретными и непрерывными уравнениями Пенлеве, ТМФ, 2000, том 122, номер 1, 5-22

DOI: https://doi.org/10.4213/tmf551

Использование Общероссийского математического портала Math-Net.Ru подразумевает, что вы прочитали и согласны с пользовательским соглашением

http://www.mathnet.ru/rus/agreement

Параметры загрузки:

IP: 3.85 .73 .92

26 апреля 2023 г., 18:16:18 
ТЕОРЕТИЧЕСКАЯ

И МАТЕМАТИЧЕСКАЯ

ФИЗИКА

Том 122, № 1

январь, 2000

(C) 2000 г. П.А. Кларксон*, Э. Л. Менсфилд ${ }^{\dagger}$, Х. Н. Вебстер ${ }^{\ddagger}$

\title{
О СООТНОШЕНИИ МЕЖДУ ДИСКРЕТНЫМИ И НЕПРЕРЫВНЫМИ УРАВНЕНИЯМИ ПЕНЛЕВЕ
}

\begin{abstract}
Обсуждается один метод вывода дифференциальных уравнений, в частности дискретных уравнений Пенлеве, из преобразований Беклунда для непрерывных уравнений Пенлеве. Используя эту технику, можно вывести первое и второе дискретные уравнения Пенлеве и некоторые из их альтернативных версий. Известно, что уравнения Пенлеве обладают иерархиями рациональных решений и однопараметрических семейств решений, выражающихся через классические специальные функции при некоторых определенных значениях параметров. Поэтому с помощью вышеупомянутых соотношений можно получить иерархии точных решений присоединенных дискретных уравнений Пенлеве. Таким образом, точные решения уравнений Пенлеве одновременно удовлетворяют дифференциальному и разностному уравнениям по аналогии со специальными функциями.
\end{abstract}

\section{1. ВВЕДЕНИЕ}

На рубеже столетий Пенлеве и его коллеги при изучении одной задачи, сформулированной Пикаром [1], исследовали новые уравнения. Пикар поставил вопрос о том, какие обыкновенные дифференциальные уравнения второго порядка вида

$$
w^{\prime \prime}=F\left(z, w, w^{\prime}\right) \quad\left({ }^{\prime} \equiv \mathrm{d} / \mathrm{d} z\right),
$$

где функция $F$ полиномиальна по $w^{\prime}$, рациональна по $w$ и аналитична по $z$, обладают тем свойством, что отличные от полюсов особые точки их любого решения зависят только от рассматриваемого уравнения и не зависят от постоянных интегрирования. Теперь это называется свойством Пенлеве. Пенлеве и его коллеги показали, что сушествуют пятьдесят канонических уравнений вида (1.1), имеющих это свойство. Шесть из них это новые нелинейные обыкновенные дифференциальные уравнения, а остальные сорок четыре либо могут быть сведены к одному из этих шести, либо могут быть проинтегрированы с помошью известных функций (ср. [2]). Эти шесть новых уравнений называются уравнениями Пенлеве и обозначаются PI-PVI.

*Institute of Mathematics and Statistics, University of Kent, Canterbury, UK. E-mail: P.A.Clarkson@ukc.ac.uk

${ }^{\dagger}$ Institute of Mathematics and Statistics, University of Kent, Canterbury, UK. E-mail: E.L.Mansfield@ukc.ac.uk

${ }^{\ddagger}$ Institute of Mathematics and Statistics, University of Kent, Canterbury, UK. Current address: Meteorological Office, London Road, Bracknell, RG12 2SZ, UK. E-mail: hnwebster@meto.gov.uk 
Хотя уравнения Пенлеве были найдены на основании чисто математических соображений, они возникают во многих физических ситуациях. По-видимому, наиболее сушественным проявлением этого является редукция уравнений в частных производных, так называемых солитонных уравнений, разрешимых с помошью метода обратной задачи теории рассеяния. Поэтому уравнения Пенлеве привлекают внимание исследователей и широко изучаются (см. [3] и приведенные там ссылки).

Уравнения Пенлеве можно интерпретировать как нелинейные аналоги уравнений для классических специальных функций. Их общие решения трансцендентны в том смысле, что они не выражаются через известные элементарные функции. Однако они имеют много рациональных решений и однопараметрических семейств решений, выражающихся через специальные функции, такие как функции Бесселя, при определенных значениях параметров (например, см. [4-17]). Далее, уравнения Пенлеве обладают преобразованиями Беклунда, связываюшими одно решение с другим либо для одного и того же уравнения при разных значениях параметров, либо для разных уравнений (см., например, $[4,5,11-21])$. Для изучения уравнений Пенлеве был разработан метод изомонодромии [22-24], и в этом смысле говорят, что они интегрируемы. Уравнения Пенлеве обладают также множеством других замечательных свойств.

С недавнего времени сушественный интерес проявляется к интегрируемым отображениям и дискретным системам. Дискретные уравнения Пенлеве dPI-dPVI стали привлекать внимание по этой причине, а также ввиду важности непрерывных уравнений Пенлеве. Дискретные уравнения Пенлеве имеют вид

$$
x_{n+1}=\frac{f_{1}\left(x_{n}, n\right)+x_{n-1} f_{2}\left(x_{n}, n\right)}{f_{3}\left(x_{n}, n\right)+x_{n-1} f_{4}\left(x_{n}, n\right)},
$$

где $f_{j}\left(x_{n}, n\right)$ - многочлены от $x_{n}$ степени не выше четырех. Дискретные уравнения Пенлеве возникают в теории ортогональных полиномов (ср. [25]), а также встречаются во многих физических приложениях, например $\mathrm{dPI}$ и $\mathrm{dPII}$ появляются в теории двумерной квантовой гравитации [26-28]. Дискретные уравнения Пенлеве dPIII-dPV выводились с помошью метода ограничения особенностей $[29,30]$, связьваюшего интегрируемый характер дискретных уравнений со структурой их особенностей. Известно, однако, что само по себе это свойство недостаточно для интегрируемости (ср. [31]). Джимбо и Сакаи [32] (см. также [33]) нашли двухкомпонентную форму dPVI, а Грамматикос и Рамани [34] недавно предложили однокомпонентную форму dPVI. В непрерывном пределе дискретные уравнения Пенлеве порождают соответствуюшие непрерывные уравнения, хотя некоторые дискретные уравнения Пенлеве дают в пределе более чем одно уравнение Пенлеве. Более того, они обладают также и множеством других общих свойств со своими непрерывными аналогами. Так, например, у них имеются лаксовы пары, билинейные представления, преобразования Беклунда и частные решения, выражаюшиеся при определенных значениях параметров через рациональные или дискретные специальные функции (см., например, [33, 35-55]).

Впрочем, дискретные уравнения Пенлеве в некотором отношении значительно богаче, чем уравнения Пенлеве. У них, например, есть некоторые решения, не имеюшие непрерывного предела, и ряд других их свойств также теряется в непрерывном пределе. Наиболее принципиальное различие состоит, по-видимому, в том, что уравнения Пенлеве имеют единственную каноническую форму, в то время как для дискретных уравнений 
Пенлеве это не так. У каждого дискретного уравнения Пенлеве имеется несколько таких различных форм. Далее, существуют два различных типа дискретных уравнений Пенлеве, а именно уравнения разностного типа, в которых независимая переменная $n$ появляется аддитивным образом, и мультипликативные $q$-дискретные уравнения Пенлеве, у которых независимая переменная $n$ возникает мультипликативным образом.

Недавно появилось несколько исследований по выводу дискретных уравнений Пенлеве из преобразований Беклунда для уравнений Пенлеве, в частности работы Фокаса, Грамматикоса и Рамани [56] (см. также [39, 46, 57, 58]), которые использовали подход, основанный на преобразованиях Шлезингера, относяшихся к присоединенной задаче изомонодромии для уравнения Пенлеве, и Громака и Цегельника [59-62], хотя последние не идентифицировали уравнения, которые они получили, как дискретные уравнения Пенлеве.

\section{2. ВЫВОД ДИСКРЕТНЫХ УРАВНЕНИЙ ПЕНЛЕВЕ ИЗ ПРЕОБРАЗОВАНИЙ БЕКЛУНДА ДЛЯ НЕПРЕРЫВНЫХ УРАВНЕНИЙ ПЕНЛЕВЕ}

Прежде чем описывать процедуру для уравнений Пенлеве, мы рассмотрим классические специальные функции, используя функции Бесселя в качестве примера.

ПримеР 2.1. Функции Бесселя. Функция Бесселя $J_{n}(z)$ удовлетворяет уравнению

$$
z^{2} J_{n}^{\prime \prime}(z)+z J_{n}^{\prime}(z)+\left(z^{2}-n^{2}\right) J_{n}(z)=0
$$

и рекуррентным соотношениям

$$
\begin{aligned}
& z J_{n}^{\prime}(z)=z J_{n-1}(z)-n J_{n}(z) \\
& z J_{n}^{\prime}(z)=n J_{n}(z)-z J_{n+1}(z) \\
& z J_{n+1}(z)-2 n J_{n}(z)+z J_{n-1}(z)=0
\end{aligned}
$$

(ср. [63]). Исключение $J_{n}^{\prime}(z)$ из (2.2) и (2.3) дает рекуррентное соотношение (2.4). Полагая $n \rightarrow n+1$ в (2.2) и исключая $J_{n+1}(z)$ с помощью (2.3), мы приходим к дифференциальному уравнению (2.1). Таким образом, функция Бесселя $J_{n}(z)$ удовлетворяет и дифференциальному уравнению (2.1), и разностному уравнению (2.4). Теперь мы покажем, что аналогичная ситуация имеет место и для уравнений Пенлеве.

Обшая процедура вывода дискретных уравнений Пенлеве из преобразований Беклунда для уравнений Пенлеве заключается в следуюшем. Предположим, что имеются два преобразования Беклунда для заданного уравнения Пенлеве вида

$$
\begin{aligned}
w^{+}\left(z ; \boldsymbol{\alpha}^{+}\right) & =\mathbf{T}^{+}(w(z ; \boldsymbol{\alpha}))=F\left(w(z ; \boldsymbol{\alpha}), w^{\prime}(z ; \boldsymbol{\alpha}), z, \boldsymbol{\alpha}\right), \\
w^{-}\left(z ; \boldsymbol{\alpha}^{-}\right) & =\mathbf{T}^{-}(w(z ; \boldsymbol{\alpha}))=G\left(w(z ; \boldsymbol{\alpha}), w^{\prime}(z ; \boldsymbol{\alpha}), z, \boldsymbol{\alpha}\right),
\end{aligned}
$$

где $w(z ; \boldsymbol{\alpha})$ - решение присоединенного уравнения Пенлеве, соответствуюшего параметрам $\boldsymbol{\alpha}=\left(\alpha_{1}, \alpha_{2}, \ldots, \alpha_{m}\right)$, а $w^{ \pm}\left(z ; \boldsymbol{\alpha}^{ \pm}\right)$- решения, соответствуюшие параметрам $\boldsymbol{\alpha}^{ \pm}=\left(\alpha_{1}^{ \pm}, \alpha_{2}^{ \pm}, \ldots, \alpha_{m}^{ \pm}\right)$. Исключение $w^{\prime}(z ; \boldsymbol{\alpha})$ из $(2.5)$ и (2.6) приводит к алгебраическому соотношению, являюшемуся рекуррентным соотношением, для трех решений $w^{+}\left(z ; \boldsymbol{\alpha}^{+}\right), w(z ; \boldsymbol{\alpha})$ и $w^{-}\left(z ; \boldsymbol{\alpha}^{-}\right)$уравнения Пенлеве. Это алгебраическое соотношение 
можно интерпретировать как нелинейный принцип суперпозиции для решений уравнения Пенлеве, как альтернативную форму преобразований Беклунда или как дискретное уравнение типа Пенлеве. Непрерьвная переменная $z$ входит в уравнение просто как параметр, а независимая переменная в дискретном уравнении порождается параметрами $\boldsymbol{\alpha}$.

Чтобы последовательно выполнить эту процедуру для заданного уравнения Пенлеве, нам потребуются два преобразования Беклунда $\mathbf{T}^{+}$и $\mathbf{T}^{-}$такие, что $\mathbf{T}^{+}=\left(\mathbf{T}^{-}\right)^{-1}$, т.е. они должны являться взаимно обратными преобразованиями, и тогда рассматриваемые решения и параметры будут связаны следуюшим образом:

$$
\left\{w^{-}\left(z ; \boldsymbol{\alpha}^{-}\right), \boldsymbol{\alpha}^{-}\right\} \underset{\mathbf{T}^{-}}{\stackrel{\mathbf{T}^{+}}{\rightleftarrows}}\{w(z ; \boldsymbol{\alpha}), \boldsymbol{\alpha}\} \underset{\mathbf{T}^{-}}{\stackrel{\mathbf{T}^{+}}{\rightleftarrows}}\left\{w^{+}\left(z ; \boldsymbol{\alpha}^{+}\right), \boldsymbol{\alpha}^{+}\right\} .
$$

Следовательно, полагая $w(z ; \boldsymbol{\alpha})=x_{n}, w^{ \pm}\left(z ; \boldsymbol{\alpha}^{ \pm}\right)=x_{n \pm 1}$, где $\boldsymbol{\alpha}=\mathbf{a}_{n}$ и $\boldsymbol{\alpha}^{ \pm}=\mathbf{a}_{n \pm 1}$, мы получаем цепочку

$$
\left\{x_{n-1}, \mathbf{a}_{n-1}\right\} \underset{\mathbf{T}^{-}}{\stackrel{\mathbf{T}^{+}}{\rightleftarrows}}\left\{x_{n}, \mathbf{a}_{n}\right\} \underset{\mathbf{T}^{-}}{\stackrel{\mathbf{T}^{+}}{\rightleftarrows}}\left\{x_{n+1}, \mathbf{a}_{n+1}\right\} .
$$

Проиллюстрируем эту процедуру на примере второго уравнения Пенлеве (см. также [56]).

Пример 2.2. Второе уравнение Пенлеве. Допустим, что $w(z ; \alpha)$ является решением второго уравнения Пенлеве РІІ

$$
w^{\prime \prime}=2 w^{3}+z w+\alpha,
$$

где $\alpha$ - константа. Известно, что в этом случае $($ ср. $[18,64])$

$$
\begin{aligned}
\mathbf{S}: & w(z ;-\alpha)=-w(z ; \alpha), \\
\mathbf{T}^{+}: & w(z ; \alpha+1)=-w(z ; \alpha)-\frac{2 \alpha+1}{2 w^{2}(z ; \alpha)+2 w^{\prime}(z ; \alpha)+z}, \quad \alpha \neq-\frac{1}{2},
\end{aligned}
$$

также решения PII (2.7) соответственно для параметров $-\alpha$ и $\alpha+1$. Комбинация этих преобразований дает преобразование Беклунда

$$
\mathbf{T}^{-}: \quad w(z ; \alpha-1)=-w(z ; \alpha)-\frac{2 \alpha-1}{2 w^{2}(z ; \alpha)-2 w^{\prime}(z ; \alpha)+z}, \quad \alpha \neq \frac{1}{2},
$$

которое является обратным к $\mathbf{T}^{+}$. Поэтому исключение $w^{\prime}(z ; \alpha)$ из $(2.9)$ и $(2.10)$ дает

$$
\frac{2 \alpha+1}{w(z ; \alpha+1)+w(z ; \alpha)}+\frac{2 \alpha-1}{w(z ; \alpha)+w(z ; \alpha-1)}+4 w^{2}(z ; \alpha)+2 z=0 .
$$

Теперь, если положить

$$
a_{n+1}=\alpha+1, \quad a_{n}=\alpha, \quad a_{n-1}=\alpha-1,
$$

а затем решить разностные уравнения, то получится

$$
\alpha=a_{n}=n+\kappa-\frac{1}{2},
$$


где $\kappa$-произвольная постоянная (множитель $1 / 2$ вводится для удобства). Так как преобразования $\mathbf{T}^{+}{ }_{\text {и }} \mathbf{T}^{-}$взаимно обратны, уравнения (2.12) имеют одно и то же общее решение. Далее, полагая $x_{m}=w\left(z ; a_{m}\right)$ в $(2.11)$, получаем разностное уравнение

$$
\frac{n+\kappa}{x_{n+1}+x_{n}}+\frac{n-1+\kappa}{x_{n}+x_{n-1}}+2 x_{n}^{2}+z=0
$$

где $z$-параметр, которое является альтернативной формой $\mathrm{dPI}(\mathrm{a}-\mathrm{dPI})[56,57]$.

Поэтому решения РІІ (2.7) удовлетворяют и дифференциальному уравнению, а именно самому уравнению PII и разностному уравнению а-dPI (2.13), что аналогично ситуации с функциями Бесселя, которая обсуждалась в предыдущем примере.

2.1. Рациональные решения. При $\kappa=1 / 2, x_{0}=0$ и $x_{1}=-1 / z$ мы получаем иерархию совместных рациональных решений PII (2.7) и а-dPI (2.13), приведенную в табл. 1.

Таблица 1

Рациональные решения PII и a-dPI

\begin{tabular}{|c|c|c|}
\hline$n$ & $\alpha$ & $x_{n}=w(z ; \alpha)$ \\
\hline 0 & 0 & 0 \\
1 & 1 & $-\frac{1}{z}$ \\
2 & 2 & $-\frac{2\left(z^{3}-2\right)}{z\left(z^{3}+4\right)}$ \\
3 & 3 & $3 z^{2}\left(z^{6}+8 z^{3}+160\right)$ \\
4 & 4 & $-\frac{4\left(z^{15}+50 z^{12}+100 z^{9}-22400 z^{6}-112000 z^{3}-224000\right.}{\left(z^{3}+4\right)\left(z^{6}+20 z^{3}-80\right)}$ \\
\hline
\end{tabular}

2.2. Решения Эйри. При $\kappa=1, x_{-1}=\Phi(z)=\phi^{\prime}(z) / \phi(z)$ и $x_{0}=-\Phi(z)$, где $\phi(z)$ удовлетворяет уравнению Эйри

$$
\phi^{\prime \prime}+\frac{1}{2} z \phi=0
$$

мы получаем иерархию рациональных решений РІІ (2.7) и а-dPI (2.13) (табл. 2). 
Решения Эйри для PII и а-dPI

\begin{tabular}{|c|c|c|}
\hline$n$ & $\alpha$ & $x_{n}=w(z ; \alpha)$ \\
\hline 0 & $\frac{1}{2}$ & $-\Phi(z)$ \\
1 & $\frac{3}{2}$ & $\frac{2 \Phi^{3}(z)+z \Phi(z)-1}{2 \Phi^{2}(z)+z}$ \\
2 & $\frac{5}{2}$ & $\frac{4 z \Phi^{4}(z)+6 \Phi^{3}(z)+4 z^{2} \Phi^{2}(z)+3 z \Phi(z)+z^{3}-1}{\left[2 \Phi^{2}(z)+z\right]}$ \\
\hline
\end{tabular}

ПримеР 2.3. Второе уравнение Пенлеве и Р34. В этом примере мы выведем систему дискретных уравнений. Пусть $v(z ; \alpha)$ и $w(z ; \alpha)$ - соответственно решения второго уравнения Пенлеве РІІ и Р 34 :

$$
\begin{aligned}
& v^{\prime \prime}=2 v^{3}+z v+\alpha, \\
& w^{\prime \prime}+2 w^{2}+z w+\frac{\left(\alpha+\frac{1}{2}\right)^{2}-w^{2}}{2 w}=0 .
\end{aligned}
$$

Название уравнения Р34 (2.15) объясняется тем, что оно эквивалентно уравнению XXXIV в главе 14 книги Айнса [2]. Известно, что [18] решения PII (2.14) и Р34 (2.15) связаны следуюшим обратимым преобразованием:

$$
\begin{aligned}
& v(z ; \alpha)=\frac{2 w^{\prime}(z ; \alpha)+2 \alpha+1}{4 w(z ; \alpha)} \\
& w(z ; \alpha)=-v^{\prime}(z ; \alpha)-v^{2}(z ; \alpha)-\frac{1}{2} z,
\end{aligned}
$$

и, более того, преобразования Беклунда для РІІ и Р34 имеют соответственно вид

$$
\begin{aligned}
& v(z ; \alpha+1)=-v(z ; \alpha)-\frac{2 \alpha+1}{2 v^{2}(z ; \alpha)+2 v^{\prime}(z ; \alpha)+z} \\
& w(z ; \alpha+1)=-w(z ; \alpha)-\left(\frac{2 w^{\prime}(z ; \alpha)-2 \alpha-1}{w(z ; \alpha)}\right)^{2} .
\end{aligned}
$$

Исключение $v^{\prime}$ из $(2.17)$ и (2.18) дает

$$
v(z ; \alpha+1)=-v(z ; \alpha)+\frac{2 \alpha+1}{2 w(z ; \alpha)},
$$

а исключение $w^{\prime}$ из (2.16) и (2.19) приводит к

$$
w(z ; \alpha+1)=-w(z ; \alpha)-z-2\left(v(z ; \alpha)-\frac{2 \alpha+1}{2 w(z ; \alpha)}\right)^{2} .
$$


Как и в предыдушем примере, решение разностных уравнений для соотношений между параметрами дает $\alpha=a_{n}=n+\kappa-1 / 2$. Итак, если положить $x_{m}=v\left(z ; a_{n}\right)$ и $y_{m}=$ $w\left(z ; a_{m}\right)$ в $(2.20)$ и $(2.21)$, то получится дискретная система

$$
\begin{aligned}
& x_{n+1}=-x_{n}+\frac{n+\kappa}{y_{n}}, \\
& y_{n+1}=-y_{n}-z-2\left(x_{n}-\frac{n+\kappa}{y_{n}}\right)^{2},
\end{aligned}
$$

где $z$-параметр.

Теперь мы рассмотрим систему (2.22). Исключая $y_{n}$, получаем dPI (2.13), а исключение $x_{n}$ приводит к уравнению второго порядка и второй степени:

$$
\frac{\left(y_{n+1}-y_{n-1}\right)^{2} y_{n}^{2}}{4(n+\kappa)^{2}}+y_{n+1}+2 y_{n}+y_{n-1}+2 z+\frac{(n+\kappa)^{2}}{y_{n}^{2}}=0 .
$$

Можно непосредственно получить это дискретное уравнение из преобразования Беклунда для Р 34 , используя присоединенное обратное преобразование

$$
w(z ; \alpha-1)=-w(z ; \alpha)-2\left(\frac{w^{\prime}(z ; \alpha)+\alpha}{2 w(z ; \alpha)-z}\right)^{2}
$$

которое легко находится из (2.19) и дискретной симметрии $w(z ;-1-\alpha)=w(z ; \alpha)$.

Таким образом, мы обобшили вышеупомянутую процедуру и получили дискретную систему (2.22). Эта система включает дискретные уравнения, получающиеся из преобразований Беклунда для РІІ и Р34, т.е. соответственно (2.13) и (2.23).

\section{3. ДИСКРЕТНЫЕ УРАВНЕНИЯ, ПОЛУЧАЮШИЕСЯ ИЗ ПРЕОБРАЗОВАНИЙ БЕКЛУНДА ДЛЯ ТРЕТЬЕГО УРАВНЕНИЯ ПЕНЛЕВЕ}

В этом разделе обсуждаются дискретные уравнения, получаюшиеся из преобразований Беклунда для третьего уравнения Пенлеве РІІІ

$$
w^{\prime \prime}=\frac{\left(w^{\prime}\right)^{2}}{w}-\frac{w^{\prime}}{z}+\frac{\alpha w^{2}+\beta}{z}+\gamma w^{3}-\frac{\delta}{w},
$$

где $\alpha, \beta, \gamma$ и $\delta$ - произвольные постоянные.

3.1. Преобразования Беклунда для PIII при $\gamma \delta \neq 0$. В общем случае, когда $\gamma \delta \neq 0$ в (3.1), мы полагаем, не нарушая обшности, что $\gamma=1$ и $\delta=-1$. Известно, что 
все преобразования Беклунда для РІІІ при $\gamma=1$ и $\delta=-1$ являются комбинациями следующих шести преобразований (дальнейшие подробности см. в [11]):

$$
\begin{array}{lll}
\mathbf{T}_{1}: w_{1}\left(z ; \alpha_{1}, \beta_{1}, 1,-1\right)=\frac{w\left(z w^{\prime}+z w^{2}+\alpha w+w+z\right)}{z w^{\prime}+z w^{2}-\beta w-w+z}, & \alpha_{1}=-\beta-2, & \beta_{1}=-\alpha-2, \\
\mathbf{T}_{2}: w_{2}\left(t ; \alpha_{2}, \beta_{2}, 1,-1\right)=\frac{w\left(z w^{\prime}-z w^{2}-\alpha w+w+z\right)}{z w^{\prime}-z w^{2}-\beta w-w+z}, & \alpha_{2}=\beta+2, & \beta_{2}=\alpha-2, \\
\mathbf{T}_{3}: w_{3}\left(z ; \alpha_{3}, \beta_{3}, 1,-1\right)=\frac{w\left(z w^{\prime}+z w^{2}+\alpha w+w-z\right)}{z w^{\prime}+z w^{2}+\beta w-w-z}, & \alpha_{3}=\beta-2, & \beta_{3}=\alpha+2, \\
\mathbf{T}_{4}: w_{4}\left(z ; \alpha_{4}, \beta_{4}, 1,-1\right)=\frac{w\left(z w^{\prime}-z w^{2}-\alpha w+w-z\right)}{z w^{\prime}-z w^{2}+\beta w-w-z}, & \alpha_{4}=-\beta+2, & \beta_{4}=-\alpha+2, \\
\widehat{\mathbf{T}}: \widehat{w}(t ; \widehat{\alpha}, \widehat{\beta}, 1,-1)=-w, & \widehat{\alpha}=-\alpha, & \widehat{\beta}=-\beta, \\
\widetilde{\mathbf{T}}: \widetilde{w}(t ; \widetilde{\alpha}, \widetilde{\beta}, 1,-1)=\frac{1}{w}, & \widetilde{\alpha}=-\beta, & \widetilde{\beta}=-\alpha,
\end{array}
$$

где $w \equiv w(z ; \alpha, \beta, 1,-1)$ - решение $(3.1)$.

Используя преобразования Беклунда $\left\{\mathbf{T}_{j}, \widehat{\mathbf{T}}_{j} \equiv \widehat{\mathbf{T}} \circ \mathbf{T}_{j}, \widetilde{\mathbf{T}}_{j} \equiv \widetilde{\mathbf{T}} \circ \mathbf{T}_{j}, \mathbf{T}_{j}^{*} \equiv \widehat{\mathbf{T}} \circ \widetilde{\mathbf{T}} \circ\right.$ $\left.\mathbf{T}_{j}\right\}_{j=1}^{4}$ для PIII, можно непосредственно показать, что существуют двенадцать совместных пар параметров $\alpha$ и $\beta$ с соответствуюшими нетривиальными решениями. $\mathrm{B}$ каждом случае, чтобы получить дискретное уравнение, нужно решить рекуррентные соотношения для $\alpha$ и $\beta$, исключить $w^{\prime}$ и подставить $\alpha$ и $\beta$.

СлучАй 3.1. $\left\{\mathbf{T}_{1}, \mathbf{T}_{1}^{*}\right\}$. Полагая $a_{n+1}=a_{1}, a_{n}=\alpha, a_{n-1}=a_{1}^{*}, b_{n+1}=b_{1}, b_{n}=\beta$, $b_{n-1}=b_{1}^{*}$ в соотношениях для параметров, мы получаем системы разностных уравнений

$$
\begin{aligned}
& a_{n+1}=-b_{n}-2, \quad a_{n-1}=-a_{n}-2, \\
& b_{n+1}=-a_{n}-2, \quad b_{n-1}=-b_{n}-2
\end{aligned}
$$

с одним и тем же общим решением

$$
a_{n}=\mu(-1)^{n}-1, \quad b_{n}=\mu(-1)^{n}-1,
$$

где $\mu$ - произвольная постоянная. Следовательно, полагая $x_{m}=w\left(z ; a_{m}, b_{m}\right)$,

$$
\begin{aligned}
& x_{n+1}=w_{1}\left(z ; a_{n+1}, b_{n+1}\right)=\frac{w\left(z w^{\prime}+z w^{2}+a_{n} w+w+z\right)}{z w^{\prime}+z w^{2}-b_{n} w-w+z} \\
& x_{n-1}=w_{1}^{*}\left(z ; a_{n-1}, b_{n-1}\right)=-\frac{\left(z w^{\prime}+z w^{2}-b_{n} w-w+z\right)}{w\left(z w^{\prime}+z w^{2}+a_{n} w+w+z\right)}
\end{aligned}
$$

и исключая $w^{\prime}$, мы получаем простое разностное уравнение

$$
x_{n+1} x_{n-1}=-1 \text {. }
$$

Применение этой процедуры к парам преобразований Беклунда $\left\{\mathbf{T}_{2}, \widetilde{\mathbf{T}}_{2}\right\},\left\{\mathbf{T}_{3}, \widetilde{\mathbf{T}}_{3}\right\}$ и $\left\{\mathbf{T}_{4}, \mathbf{T}_{4}^{*}\right\}$ тоже дает разностные уравнения, которые эквивалентны (3.2). 
СлучАй 3.2. $\left\{\widehat{\mathbf{T}}_{1}, \widehat{\mathbf{T}}_{4}\right\}$. Полагая $a_{n+1}=\widehat{a}_{1}, a_{n}=\alpha, a_{n-1}=\widehat{a}_{4}, b_{n+1}=\widehat{b}_{1}, b_{n}=\beta$, $b_{n-1}=\widehat{b}_{4}$ в соотношениях для параметров, мы получаем системы разностных уравнений

$$
\begin{aligned}
a_{n+1}=b_{n}+2, & a_{n-1}=b_{n}-2, \\
b_{n+1}=a_{n}+2, & b_{n-1}=a_{n}-2
\end{aligned}
$$

с одним и тем же обшим решением

$$
a_{n}=\kappa+\mu(-1)^{n}+2 n, \quad b_{n}=\kappa-\mu(-1)^{n}+2 n,
$$

где $\kappa$ и $\mu$ - произвольные постоянные. Поэтому подстановка $x_{m}=w\left(z ; a_{m}, b_{m}\right)$,

$$
\begin{aligned}
& x_{n+1}=\widehat{w}_{1}\left(z ; a_{n+1}, b_{n+1}\right)=-\frac{w\left(z w^{\prime}+z w^{2}+a_{n} w+w+z\right)}{z w^{\prime}+z w^{2}-b_{n} w-w+z}, \\
& x_{n-1}=\widehat{w}_{4}\left(z ; a_{n-1}, b_{n-1}\right)=-\frac{w\left(z w^{\prime}-z w^{2}-a_{n} w+w-z\right)}{z w^{\prime}-z w^{2}+b_{n} w-w-z}
\end{aligned}
$$

и исключение $w^{\prime}$ дают

$$
\frac{\kappa+2 n+1}{x_{n+1}+x_{n}}+\frac{\kappa+2 n-1}{x_{n}+x_{n-1}}+z-\frac{2 n+\kappa-\mu(-1)^{n}}{x_{n}}+\frac{z}{x_{n}^{2}}=0 .
$$

Можно показать, что континуальный предел этого уравнения приводит к частному случаю PIII (3.1) с $\alpha=\beta$ и $\gamma=\delta=0$. Применение этой процедуры к паре преобразований Беклунда $\left\{\widehat{\mathbf{T}}_{2}, \widehat{\mathbf{T}}_{3}\right\}$ также дает разностное уравнение, которое эквивалентно (3.3).

СлучАй 3.3. $\left\{\mathbf{T}_{2}^{*}, \mathbf{T}_{3}^{*}\right\}$. Полагая $a_{n+1}=a_{2}^{*}, a_{n}=\alpha, a_{n-1}=a_{3}^{*}, b_{n+1}=b_{2}^{*}, b_{n}=$ $\beta, b_{n-1}=b_{3}^{*}$ в соотношениях для параметров, мы получаем системы разностных уравнений

$$
\begin{aligned}
& a_{n+1}=a_{n}-2, \quad a_{n-1}=a_{n}+2, \\
& b_{n+1}=b_{n}+2, \quad b_{n-1}=b_{n}-2
\end{aligned}
$$

с одним и тем же общим решением

$$
a_{n}=2 \kappa-2 n, \quad b_{n}=2 \mu+2 n,
$$

где $\kappa$ и $\mu$-произвольные постоянные. Следовательно, подставляя $x_{m}=w\left(z ; a_{m}, b_{m}\right)$,

$$
\begin{aligned}
& x_{n+1}=w_{2}^{*}\left(z ; a_{n+1}, b_{n+1}\right)=-\frac{\left(z w^{\prime}-z w^{2}-b_{n} w-w+z\right)}{w\left(z w^{\prime}-z w^{2}-a_{n} w+w+z\right)}, \\
& x_{n-1}=w_{3}^{*}\left(z ; a_{n-1}, b_{n-1}\right)=-\frac{\left(z w^{\prime}+z w^{2}+b_{n} w-w-z\right)}{w\left(z w^{\prime}+z w^{2}+a_{n} w+w-z\right)}
\end{aligned}
$$

и исключая $w^{\prime}$, мы приходим к

$$
\frac{2 n+1-\kappa+\mu}{x_{n} x_{n+1}+1}+\frac{2 n-1-\kappa+\mu}{x_{n} x_{n-1}+1}+x_{n} z-2 n+2 \kappa-\frac{z}{x_{n}}=0,
$$

что представляет собой альтернативную форму дляя $\mathrm{dPII}[46,56]$. Применение этой процедуры к паре преобразований Беклунда $\left\{\widetilde{\mathbf{T}}_{1}, \widetilde{\mathbf{T}}_{4}\right\}$ также дает разностное уравнение, которое эквивалентно dPII (3.4).

Поэтому непрерывные преобразования Беклунда для РІІІ приводят к двум дискретным уравнениям Пенлеве вида (3.3) и (3.4). Как и для всех дискретных уравнений Пенлеве, названия этих уравнений происходят от непрерывных уравнений, получающихся в непрерывном пределе, хотя, как было отмечено вьше, здесь нет единственности. 
3.2. Пример. Теперь мы обсудим один пример, показывающий, что совместность значений параметров сама по себе недостаточна для вывода дискретных уравнений Пенлеве. Дело в том, что надо еще проверить, являются ли преобразования Беклунда взаимно обратными.

Рассмотрим преобразования Беклунда $\widetilde{\mathbf{T}}_{1}$ и $\widehat{\mathbf{T}}_{4}$, определенные формулами

$$
\begin{aligned}
& \widetilde{\mathbf{T}}_{1}: \widetilde{w}_{1}\left(z ; \widetilde{\alpha}_{1}, \widetilde{\beta}_{1}, 1,-1\right)=\frac{z w^{\prime}+z w^{2}-\beta w-w+z}{w\left(z w^{\prime}+z w^{2}+\alpha w+w+z\right)}, \quad \widetilde{\alpha}_{1}=\alpha+2, \quad \widetilde{\beta}_{1}=\beta+2, \\
& \widehat{\mathbf{T}}_{4}: \widehat{w}_{4}\left(z ; \widehat{\alpha}_{4}, \widehat{\beta}_{4}, 1,-1\right)=-\frac{w\left(z w^{\prime}-z w^{2}-\alpha w+w-z\right)}{z w^{\prime}-z w^{2}+\beta w-w-z}, \widehat{\alpha}_{4}=\beta-2, \quad \widehat{\beta}_{4}=\alpha-2 .
\end{aligned}
$$

Полагая $a_{n+1}=\widetilde{a}_{1}, a_{n}=\alpha, a_{n-1}=\widehat{a}_{4}, b_{n+1}=\widetilde{b}_{1}, b_{n}=\beta, b_{n-1}=\widehat{b}_{4}$ в соотношениях для параметров, мы получаем системы разностных уравнений

$$
\begin{gathered}
\alpha_{n+1}=\alpha_{n}+2, \quad \alpha_{n-1}=\beta_{n}-2 \\
\beta_{n+1}=\beta_{n}+2, \quad \beta_{n-1}=\alpha_{n}-2
\end{gathered}
$$

с одним и тем же решением

$$
\alpha_{n}=\kappa+2 n, \quad \beta_{n}=\kappa+2 n,
$$

где $\kappa$ - произвольная постоянная. Заметим, что здесь имеется только одна произвольная постоянная, и поэтому эта ситуация не является столь общей, как в случаях, обсуждавшихся вьше. Следуя прежней процедуре, мы исключаем $w^{\prime}$ из преобразований Беклунда $\widetilde{\mathbf{T}}_{1}$ и $\widehat{\mathbf{T}}_{4}$ и получаем дискретное уравнение

$$
z x_{n}+1+\frac{z}{x_{n}}+\frac{x_{n}(2 n+\kappa-1)}{x_{n}+x_{n-1}}+\frac{2 n+\kappa+1}{x_{n} x_{n+1}-1}=0 .
$$

Теперь мы продолжим исследование отображения (3.5) и изучим его действие на рациональные решения PIII (3.1), в котором $\alpha=\beta, \gamma=1$ и $\delta=-1$. Применяя преобразование Беклунда $\widetilde{\mathbf{T}}_{1}$ к простому рациональному решению $w(z ; 0,0,1,-1)=1$, мы получаем рациональное решение $w(z ; 2,2,1,-1)=(2 z-1) /(2 z+1)$. Подстановка $x_{0}=1$, $x_{1}=(2 z-1) /(2 z+1)$ и $\kappa=0$ в $(3.5)$ дает выражение

$$
x_{2}=\frac{(2 z+1)\left(32 z^{4}-24 z^{3}+4 z^{2}+6 z+1\right)}{(2 z-1)\left(32 z^{4}+24 z^{3}+4 z^{2}-6 z+1\right)},
$$

не являющееся решением PIII (3.1). Поэтому (3.5) не является отображением для решений PIII.

Возникает вопрос, почему совместность параметров недостаточна в данном случае? Чтобы разобраться в этом, рассмотрим рациональное решение

$$
w(z ; 2,2,1,-1)=\frac{2 z-1}{2 z+1}
$$

входящее в так называемую “ $w=1$ ”-иерархию (см. [11]). Здесь применение преобразования Беклунда $\widetilde{\mathbf{T}}_{1}$, а затем преобразования $\widehat{\mathbf{T}}_{4}$ к $(3.6)$ дает рациональное решение

$$
w(z ; 2,2,1,-1)=-\frac{2 z+1}{2 z-1}
$$


входящее в “ $w=-1$ ”-иерархию. Следовательно, рассматриваемое затруднение вызвано неединственностью решений PIII (3.1). Заданному набору параметров может соответствовать несколько решений. Эта неединственность объясняет, почему совместность параметров недостаточна.

В обшем случае можно непосредственно показать, что $\widehat{\mathbf{T}}_{4}\left(\widetilde{\mathbf{T}}_{1}(w)\right)=-1 / w$. Значит, преобразования $\widetilde{\mathbf{T}}_{1}$ и $\widehat{\mathbf{T}}_{4}$ не являются взаимно обратными, и поэтому смысл уравнения (3.5) здесь неясен. В самом деле, мы не считаем, что это дискретное уравнение Пенлеве и что непрерывньй предел (3.5) неизвестен на этой стадии. Действительно, оказывается, что дискретное уравнение (3.5) не является интегрируемым. Чтобы показать это, рассмотрим ограничение особенностей для (3.5). Найдя $x_{n+1}$, мы видим, что расходимость может возникнуть, если $x_{n}=0$ для некоторого $n \in \mathbb{N}$. Мы полагаем $x_{n}=\varepsilon$ вместо строгого равенства нулю и по-прежнему считаем, что $x_{n-1}$ произвольно. Тогда исследование итерации (3.5) дает

$$
\begin{array}{ll}
x_{n+1}=\varepsilon^{-1}+O(1), & x_{n+3}=\varepsilon^{-1}+O(1), \\
x_{n+2}=\varepsilon+O\left(\varepsilon^{2}\right), & x_{n+4}=\varepsilon+O\left(\varepsilon^{2}\right)
\end{array}
$$

и т.д. до бесконечности. Поэтому дискретное уравнение (3.5) не обладает свойством ограничения особенностей и вследствие этого скорее всего неинтегрируемо.

3.3. Преобразования Беклунда для PIII при $\gamma=0$. Как и в общем случае, при $\gamma=0$ мы можем зафиксировать два параметра, выбирая масштабы для зависимой и независимой переменных. Если $\gamma=0$, то мы полагаем $\alpha=1$ и $\delta=-1$, не нарушая обшности, и $w(z ; \beta) \equiv w(z ; 1, \beta, 0,-1)$, и тогда $\mathrm{PIII}(3.1)$ имеет преобразования Беклунда

$$
\begin{array}{ll}
\mathbf{T}_{5}: & w_{5}\left(z ; \beta_{5}\right)=\frac{z w^{\prime}(z ; \beta)-(1+\beta) w(z ; \beta)+z}{w^{2}(z ; \beta)}, \quad \beta_{5}=\beta+2, \\
\mathbf{T}_{6}: & w_{6}\left(z ; \beta_{6}\right)=-\frac{z w^{\prime}(z ; \beta)-(1-\beta) w(z ; \beta)-z}{w^{2}(z ; \beta)}, \quad \beta_{6}=\beta-2,
\end{array}
$$

которые являются взаимно обратными. Подстановка $b_{n+1}=\beta_{5}, b_{n}=\beta$ и $b_{n-1}=\beta_{6}$ приводит к рекуррентным соотношениям $b_{n+1}=b_{n}+2$ и $b_{n-1}=b_{n}-2$, имеющим одно и то же общее решение $b_{n}=2(\kappa+n)$, где $\kappa$ - произвольная постоянная. Исключая $w^{\prime}(z ; \beta)$ из $\mathbf{T}_{5}$ и $\mathbf{T}_{6}$ и полагая затем $x_{m}=w\left(z ; b_{m}\right)$, мы получаем дискретное уравнение

$$
x_{n+1}+x_{n-1}=-\frac{4(\kappa+n)}{x_{n}}+\frac{2 z}{x_{n}^{2}},
$$

являющееся альтернативной формой dPI [56] (см. также [59]).

3.4. Преобразования Беклунда для PIII при $\delta=0$. Если $\delta=0$, мы полагаем, не нарушая обшности, что $\beta=1$ и $\gamma=1$, и тогда PIII (3.1) тоже имеет следуюшие преобразования Беклунда:

$$
\begin{array}{ll}
\mathbf{T}_{7}: & w_{7}\left(z ; \alpha_{7}\right)=-\frac{1}{z w^{\prime}(z ; \alpha)+(1+\alpha) w(z ; \alpha)+z w^{2}(z ; \alpha)}, \\
\mathbf{T}_{8}: & w_{8}\left(z ; \alpha_{8}\right)=\frac{1}{z w^{\prime}(z ; \alpha)+(1-\alpha) w(z ; \alpha)-z w^{2}(z ; \alpha)}, \quad \alpha_{8}=\alpha-2,
\end{array}
$$


где $w(z ; \alpha) \equiv w(z ; \alpha, 1,1,0)$. Подстановка $a_{n+1}=\alpha_{7}, a_{n}=\alpha$ и $a_{n-1}=\alpha_{8}$ приводит к рекуррентным соотношениям $a_{n+1}=a_{n}+2$ и $a_{n-1}=a_{n}-2$, имеюшим одно и то же обшее решение $a_{n}=2(\kappa+n)$, где $\kappa$-произвольная постоянная. Исключая $w^{\prime}(z ; \alpha)$ из $\mathbf{T}_{7}$ и $\mathbf{T}_{8}$ и полагая затем $x_{m}=w\left(z ; \alpha_{m}\right)$, мы получаем дискретное уравнение, которое эквивалентно альтернативной форме dPI (3.7).

\section{4. ДИСКРЕТНЫЕ УРАВНЕНИЯ, ПОРОЖДЕННЫЕ ПРЕОБРАЗОВАНИЯМИ БЕКЛУНДА ДЛЯ ЧЕТВЕРТОГО УРАВНЕНИЯ ПЕНЛЕВЕ}

В этом разделе мы обсуждаем дискретные уравнения, порожденные преобразованиями Беклунда для четвертого уравнения Пенлеве

$$
w^{\prime \prime}=\frac{\left(w^{\prime}\right)^{2}}{2 w}+\frac{3}{2} w^{3}+4 z w^{2}+2\left(z^{2}-\alpha\right) w+\frac{\beta}{w}
$$

где $\alpha$ и $\beta$ - произвольные постоянные.

4.1. Преобразования Беклунда для четвертого уравнения Пенлеве. Канонические преобразования Беклунда для PIV имеют вид

$$
\begin{aligned}
\widetilde{\mathbf{W}}^{ \pm}: & \widetilde{w}^{ \pm}\left(z ; \widetilde{\alpha}^{ \pm}, \widetilde{\beta}^{ \pm}\right)=\frac{w^{\prime}-w^{2}-2 z w \mp \sqrt{-2 \beta}}{2 w}, \\
& \widetilde{\alpha}^{ \pm}=\frac{1}{4}(2-2 \alpha \pm 3 \sqrt{-2 \beta}), \quad \widetilde{\beta}^{ \pm}=-\frac{1}{2}\left(1+\alpha \pm \frac{1}{2} \sqrt{-2 \beta}\right)^{2}, \\
\widehat{\mathbf{W}}^{ \pm}: & \widehat{w}^{ \pm}\left(z ; \widehat{\alpha}^{ \pm}, \widehat{\beta}^{ \pm}\right)=-\frac{w^{\prime}+w^{2}+2 z w \mp \sqrt{-2 \beta}}{2 w}, \\
& \widehat{\alpha}^{ \pm}=-\frac{1}{4}(2+2 \alpha \pm 3 \sqrt{-2 \beta}), \quad \widehat{\beta}^{ \pm}=-\frac{1}{2}\left(1-\alpha \pm \frac{1}{2} \sqrt{-2 \beta}\right)^{2}, \\
\mathbf{W}^{\dagger \pm}: & w^{\dagger \pm}\left(z ; \alpha^{\dagger \pm}, \beta^{\dagger \pm}\right)=w+\frac{2\left(1-\alpha \mp \frac{1}{2} \sqrt{-2 \beta}\right) w}{w^{\prime} \pm \sqrt{-2 \beta}+2 z w+w^{2}}, \\
& \alpha^{\dagger \pm}=\frac{3}{2}-\frac{1}{2} \alpha \mp \frac{3}{4} \sqrt{-2 \beta}, \quad \beta^{\dagger \pm}=-\frac{1}{2}\left(1-\alpha \pm \frac{1}{2} \sqrt{-2 \beta}\right)^{2}, \\
\mathbf{W}^{\ddagger \pm}: & w^{\ddagger \pm}\left(z ; \alpha^{\ddagger \pm}, \beta^{\ddagger \pm}\right)=w+\frac{2\left(1+\alpha \pm \frac{1}{2} \sqrt{-2 \beta}\right) w}{w^{\prime} \mp \sqrt{-2 \beta}-2 z w-w^{2}}, \\
& \alpha^{\ddagger \pm}=-\frac{3}{2}-\frac{1}{2} \alpha \mp \frac{3}{4} \sqrt{-2 \beta}, \quad \beta^{\ddagger \pm}=-\frac{1}{2}\left(-1-\alpha \pm \frac{1}{2} \sqrt{-2 \beta}\right)^{2},
\end{aligned}
$$

$\overline{\mathbf{W}}: \quad \bar{w}(z ; \bar{\alpha}, \bar{\beta})=-i w(i z ; \alpha, \beta), \quad \bar{\alpha}=-\alpha, \quad \bar{\beta}=\beta$,

где $w \equiv w(z ; \alpha, \beta)$ - решение PIV (4.1). Эти четыре преобразования справедливы для всех решений $w(z ; \alpha, \beta)$, у которых числители и знаменатели отличны от нуля. Преобразования Беклунда $\widetilde{\mathbf{W}}^{ \pm}$и $\widehat{\mathbf{W}}^{ \pm}$были найдены Лукашевичем [65] и Громаком [6], а $\mathbf{W}^{\dagger \pm}$ и $\mathbf{W}^{\ddagger \pm}$ - Китаевым [66] (см. также $[5,12,19]$ ). Преобразование $\mathbf{W}^{\dagger \pm}$ эквивалентно преобразованиям $\mathbf{T}_{1}$ (при нижнем знаке) и $\mathbf{T}_{2}$ (при верхнем знаке), полученным Муратой [12]. Заметим, что преобразования Беклунда $\mathbf{W}^{\dagger \pm}$ и $\mathbf{W}^{\ddagger \pm}$ можно выразить через преобразования $\widetilde{\mathbf{W}}^{ \pm}$и $\widehat{\mathbf{W}}^{ \pm}$(дальнейшие подробности см. в [5]). 
В преобразованиях Беклунда для PIV имеется трудность, вызванная членом с квадратным корнем $\sqrt{-2 \beta}$, и поэтому проше положить $\gamma=\sqrt{-2 \beta}$ и далее рассматривать уравнение

$$
w^{\prime \prime}=\frac{\left(w^{\prime}\right)^{2}}{2 w}+\frac{3}{2} w^{3}+4 z w^{2}+2\left(z^{2}-\alpha\right) w-\frac{\gamma^{2}}{2 w}
$$

вместо (4.1), хотя необходимо проявить внимание при выборе знака для $\gamma$. Из этого следует, что соотношения для параметров становятся линейными, а это весьма удобно.

СлучАй 4.1. $\left\{\widetilde{\mathbf{W}}^{+}, \widehat{\mathbf{W}}^{-}\right\}$. В этом случае соотношения для параметров дают систему разностных уравнений

$$
\begin{aligned}
a_{n+1} & =\frac{1}{2}-\frac{1}{2} a_{n}+\frac{3}{4} c_{n}, & a_{n-1} & =-\frac{1}{2}+\frac{1}{2} a_{n}-\frac{3}{4} c_{n}, \\
c_{n+1} & =1+a_{n}+\frac{1}{2} c_{n}, & c_{n-1} & =-1+a_{n}+\frac{1}{2} c_{n},
\end{aligned}
$$

имеющих одно и то же общее решение

$$
a_{n}=\frac{1}{2} \kappa-\frac{3}{2} \mu(-1)^{n}+\frac{1}{2} n, \quad c_{n}=\kappa+\mu(-1)^{n}+n
$$

где $\kappa$ и $\mu$ - произвольные постоянные. Исключая $w^{\prime}$ из $\widetilde{\mathbf{W}}^{+}$и $\widehat{\mathbf{W}}^{-}$и полагая $x_{n}=$ $w\left(z ; a_{n}, c_{n}\right), x_{n+1}=\widetilde{w}^{+}\left(z ; a_{n+1}, c_{n+1}\right)$ и $x_{n-1}=\widehat{w}^{-}\left(z ; a_{n-1}, c_{n-1}\right)$, где $c_{m}=\sqrt{-2 b_{m}}$, получаем

$$
x_{n+1}+x_{n}+x_{n-1}=-2 z+\frac{-n+\kappa(-1)^{n}+\mu}{x_{n}},
$$

что представляет собой dPI. На связь между решениями PIV (4.1) и dPI (4.3) впервые указали Фокас, Итс и Китаев [67] (см. также [56, 59, 60]). Впоследствии она была изучена Бассомом, Кларксоном и Хиксом [5, 68], которые составили таблицы совместных решений для PIV (4.1) и dPI (4.3), выраженных через функции параболического цилиндpa, а недавно - Грамматикосом и Рамани [58]. Эта связь между решениями PIV (4.1) и $\mathrm{dPI}$ (4.3) проявляется в удивительном сходстве результатов в [58] для $\mathrm{dPI}(4.3)$ и в [5, 12 , 16, 69] для PIV (4.1).

Применение этой процедуры к паре преобразований Беклунда $\left\{\widetilde{\mathbf{W}}^{-}, \widehat{\mathbf{W}}^{+}\right\}$также приводит к разностному уравнению, которое эквивалентно dPI (4.3).

СлучАй 4.2. $\left\{\overline{\mathbf{W}}^{\dagger+} \equiv \overline{\mathbf{W}} \circ \mathbf{W}^{\dagger+}, \overline{\mathbf{W}}^{\ddagger+} \equiv \overline{\mathbf{W}} \circ \mathbf{W}^{\ddagger+}\right\}$. В этом случае соотношения для параметров дают систему разностных уравнений

$$
\begin{aligned}
a_{n+1} & =-\frac{3}{2}+\frac{1}{2} a_{n}+\frac{3}{4} c_{n}, & a_{n-1} & =\frac{3}{2}+\frac{1}{2} a_{n}+\frac{3}{4} c_{n}, \\
c_{n+1} & =-1+a_{n}-\frac{1}{2} c_{n}, & c_{n-1} & =1+a_{n}-\frac{1}{2} c_{n},
\end{aligned}
$$

имеюших одно и то же общее решение

$$
a_{n}=-\frac{3}{2} \kappa+\frac{1}{2} \mu(-1)^{n}-\frac{3}{2} n, \quad c_{n}=-\kappa-\mu(-1)^{n}-n,
$$


где $\kappa$ и $\mu$-произвольные постоянные. Исключение $w^{\prime}$ из $\overline{\mathbf{W}}^{\dagger+}$ и $\overline{\mathbf{W}}^{\ddagger+}$ и подстановка $x_{n}=w\left(z ; a_{n}, c_{n}\right), x_{n+1}=\bar{w}^{\dagger+}\left(z ; a_{n+1}, c_{n+1}\right)$ и $x_{n-1}=\bar{w}^{\ddagger+}\left(z ; a_{n-1}, c_{n-1}\right)$ приводят к выражению

$$
\frac{2 n+1+2 \kappa}{x_{n+1}-x_{n}}+\frac{2 n-1+2 \kappa}{x_{n-1}-x_{n}}+x_{n}+2 z+\frac{n+\kappa+\mu(-1)^{n}}{x_{n}} .
$$

Полагая затем в этом уравнении $x_{m}=(-1)^{m} y_{m}$, мы получаем

$$
\frac{2 n+1+2 \kappa}{y_{n+1}+y_{n}}+\frac{2 n-1+2 \kappa}{y_{n}+y_{n-1}}=y_{n}+2(-1)^{n} z-\frac{n+\kappa+\mu(-1)^{n}}{y_{n}}
$$

что является еще одной альтернативной формой для $\mathrm{dPI}$.

Применение этой процедуры к паре преобразований Беклунда $\left\{\overline{\mathbf{W}}^{\dagger-} \equiv \overline{\mathbf{W}} \circ \mathbf{W}^{\dagger-}\right.$, $\left.\overline{\mathbf{W}}^{\ddagger-} \equiv \overline{\mathbf{W}} \circ \mathbf{W}^{\ddagger-}\right\}$ дает разностное уравнение, которое эквивалентно альтернативной форме dPI (4.4).

4.2. Разностное уравнение второго порядка и второй степени. Следующие два преобразования Беклунда для PIV (4.1), выведенные Фокасом, Мьюганом и Абловищем [19], записываются как преобразования $\mathbf{R}_{5}$ и $\mathbf{R}_{7}$, заданные формулами

$$
\begin{array}{ll}
\mathbf{R}_{5}: & w_{5}\left(z ; \alpha_{5}, \beta_{5}\right)=\frac{\left(w^{\prime}-w^{2}-2 z w\right)^{2}+2 \beta}{2 w\left[w^{\prime}-w^{2}-2 z w+2(\alpha+1)\right]}, \quad \alpha_{5}=\alpha+2, \quad \beta_{5}=\beta, \\
\mathbf{R}_{7}: & w_{7}\left(z ; \alpha_{7}, \beta_{7}\right)=\frac{\left(w^{\prime}+w^{2}+2 z w\right)^{2}+2 \beta}{2 w\left[w^{\prime}+w^{2}+2 z w-2(\alpha-1)\right]}, \quad \alpha_{7}=\alpha-2, \quad \beta_{7}=\beta,
\end{array}
$$

которые совпадают с преобразованиями $\mathbf{T}_{+}$и $\mathbf{T}_{-}$, найденными Муратой [12]. Заметим, что Бассом, Кларксон и Хикс [5] показали, что $\mathbf{R}_{5}$ и $\mathbf{R}_{7}$ можно выразить через преобразования $\widetilde{\mathbf{W}}^{ \pm}$и $\widehat{\mathbf{W}}^{ \pm}$. Подстановка $\alpha_{5}=a_{n+1}, \alpha=a_{n}, \alpha_{7}=a_{n-1}$ и решение разностных уравнений дают $\alpha_{n}=\kappa+2 n$, где $\kappa-$ произвольная постоянная. Полагая затем $x_{n+1}=w_{5}\left(z ; a_{n+1}, \beta\right), x_{n}=w\left(z ; a_{n}, \beta\right)$ и $x_{n-1}=w_{7}\left(z ; a_{n-1}, \beta\right)$ и исключая $w^{\prime}$, мы получаем разностное уравнение второго порядка и второй степени:

$$
\begin{aligned}
& x_{n+1}^{2}\left\{2 x_{n}^{4}+8 x_{n}^{3} z+4\left(2 z^{2}-\alpha-2 n-1\right) x_{n}^{2}-8 z(\alpha+2 n+1) x_{n}+\right. \\
& \left.\quad+2(\alpha+2 n+1)^{2}+\beta-\left[2 x_{n}^{2}-4 z x_{n}-4(\alpha+2 n)\right] x_{n} x_{n-1}\right\}+ \\
& \quad+2 x_{n}^{6}+4 x_{n}^{5}\left(4 z+x_{n+1}-x_{n-1}\right)+24 z x_{n}^{4}\left(x_{n+1}-x_{n-1}\right)-6 x_{n}^{4} x_{n+1} x_{n-1}+ \\
& \quad+48 z^{2} x_{n}^{3}\left(x_{n+1}-x_{n-1}\right)-4 x_{n}^{3}\left(x_{n-1}+x_{n+1}\right)-4(\alpha+2 n) x_{n}^{3}\left(x_{n+1}-x_{n-1}\right)- \\
& \quad-24 z x_{n}^{3} x_{n+1} x_{n-1}+8\left(\alpha+2 n-3 z^{2}\right) x_{n}^{2} x_{n+1} x_{n-1}+ \\
& \quad+16 z(\alpha+2 n) x_{n} x_{n+1} x_{n-1}+\left[4(\alpha+2 n)^{2}-4-2\right] x_{n+1} x_{n-1}- \\
& \quad-16 z\left(\alpha+2 n+1-2 z^{2}\right) x_{n}^{2} x_{n+1}+16 z\left(\alpha+2 n-1-2 z^{2}\right) x_{n}^{2} x_{n-1}- \\
& \quad-16 z^{2}(\alpha+2 n+1) x_{n} x_{n+1}+16 z^{2}(\alpha+2 n-1) x_{n} x_{n-1}+ \\
& \quad+48 z^{2} x_{n}^{4}+64 z^{3} x_{n}^{3}+32 z^{4}+4\left(x_{n}+2 z\right)^{2}+4\left(x_{n}+2 z\right)\left(x_{n+1}-x_{n-1}\right)+ \\
& \quad+x_{n-1}^{2}\left\{2 x_{n}^{4}+8 x_{n}^{3} z+4\left(2 z^{2}-\alpha-2 n+1\right) x_{n}^{2}-8 z(\alpha+2 n-1) x_{n}+\right. \\
& \left.\quad+2(\alpha+2 n-1)^{2}+\beta+\left[2 x_{n}^{2}+4 z x_{n}-4(\alpha+2 n)\right] x_{n} x_{n+1}\right\}=0 .
\end{aligned}
$$


4.3. Дробные преобразования Китаева. Китаев [66] исследовал преобразования Беклунда для PIV (4.1) с помошью представления ассоциированной деформации изомонодромии, которое имеет регулярную особую точку при $\lambda=0$ и нерегулярную особую точку ранга 2 при $\lambda=\infty$ (ср. [19]). Китаев показал, что преобразования Беклунда $\widetilde{\mathbf{W}}$ и $\widehat{\mathbf{W}}$ соответствуют регулярной точке при $\lambda=0$, а преобразования $\mathbf{W}^{\dagger}$ и $\mathbf{W}^{\ddagger}$ связаны с нерегулярной точкой при $\lambda=\infty$. Как было отмечено выше, Бассом, Кларксон и Хикс [5] показали,что преобразования Беклунда $\mathbf{W}^{\dagger \pm}$ и $\mathbf{W}^{\ddagger \pm}$ выражаются через $\widetilde{\mathbf{W}}^{ \pm}$и $\widehat{\mathbf{W}}^{ \pm}$. Таким образом, они получили следуюшие дробнье преобразования Китаева:

$$
\begin{aligned}
& w^{\dagger+}\left(z ; \alpha^{\dagger+}, \beta^{\dagger+}\right)=w(z ; \alpha, \beta)-\frac{1-\alpha-\frac{1}{2} \sqrt{-2 \beta}}{\widehat{w}^{-}(z ; \alpha, \beta)}, \\
& w^{\dagger-}\left(z ; \alpha^{\dagger-}, \beta^{\dagger-}\right)=w(z ; \alpha, \beta)-\frac{1-\alpha+\frac{1}{2} \sqrt{-2 \beta}}{\widehat{w}^{+}(z ; \alpha, \beta)}, \\
& w^{\ddagger+}\left(z ; \alpha^{\ddagger+}, \beta^{\ddagger+}\right)=w(z ; \alpha, \beta)+\frac{1+\alpha+\frac{1}{2} \sqrt{-2 \beta}}{\widetilde{w}^{+}(z ; \alpha, \beta)}, \\
& w^{\ddagger-}\left(z ; \alpha^{\ddagger-}, \beta^{\ddagger-}\right)=w(z ; \alpha, \beta)+\frac{1+\alpha-\frac{1}{2} \sqrt{-2 \beta}}{\widetilde{w}^{-}(z ; \alpha, \beta)},
\end{aligned}
$$

представляюшие собой алгебраические соотношения или нелинейные формуль суперпозичии для трех решений PIV (4.1). Преобразования (4.5)-(4.8) могут быть использованы для эффективного нахождения иерархий решений для PIV c помошью одних только алгебраических операций (дальнейшие подробности см. в [5]). Интересным открытым вопросом является выяснение того, относятся ли дробные преобразования Китаева (4.5)-(4.8) к дискретным уравнениям или к их специальным случаям.

\section{5. ОБСУЖДЕНИЕ}

В этой статье мы показали, что имеется тесная связь между преобразованиями Беклунда и иерархиями решений уравнений Пенлеве и дискретных уравнений Пенлеве. Иерархии решений уравнений Пенлеве удовлетворяют как дифференциальному, так и разностному уравнению. Преобразования Беклунда уравнений Пенлеве можно применить для вывода разнообразных разностных уравнений (включая некоторые дискретные уравнения Пенлеве).

Иерархии точных решений для PII, PIII и PIV, как рациональных, так и однопараметрических семейств решений, широко известны (ср. [4-9, 11-13, 16, 17, 69-77]). Поскольку сушествует явное соотношение между PII, PIII и PIV и дискретными уравнениями Пенлеве, эти иерархии решений для PII, PIII и PIV тоже удовлетворяют не только обыкновенным дифференциальным уравнениям, но и разностным уравнениям. Это вполне аналогично ситуации с классическими специальными функциями, такими как функции Бесселя, гипергеометрические функции, функции Лежандра, Вебера-Эрмита и Уитеккера, удовлетворяюшими и обыкновенному дифференшиальному уравнению, и рекуррентному соотношению, которое является дискретным уравнением. Это еше одно свидетельство того, что уравнения Пенлеве можно интерпретировать как уравнения для нелинейных специальных функций и что существует глубокая связь между классическими специальными функциями, уравнениями Пенлеве и дискретными уравнениями 
Пенлеве (см., например, также [54]).

В заключение мы сформулируем некоторые открытые вопросы, касающиеся соотношения между дискретными и непрерывными уравнениями Пенлеве.

1. Являются ли все разностные уравнения, выведенные из преобразований Беклунда для уравнений Пенлеве, “интегрируемыми”? Заметим, что лаксова пара для уравнения Пенлеве принимает форму

$$
\frac{\partial \Psi}{\partial \zeta}=\mathbf{A} \Psi, \quad \frac{\partial \Psi}{\partial z}=\mathbf{U} \Psi
$$

где $z$ - независимая переменная и переменная $\zeta$ связана с задачей Римана-Гильберта, а лаксова пара для дискретного уравнения Пенлеве имеет вид

$$
\frac{\partial \Psi_{n}}{\partial \zeta}=\mathbf{A}_{n} \Psi_{n}, \quad \Psi_{n+1}=\mathbf{U}_{n} \Psi_{n}
$$

где первое уравнение такое же, как в непрерывной задаче.

2. Как можно алгоритмически получить лаксову пару (или задачу монодромии) для дискретного уравнения из лаксовой пары (или задачи монодромии) и преобразований Беклунда для присоединеного уравнения Пенлеве?

3. Можно ли получить все дискретные уравнения Пенлеве из преобразований Беклунда для уравнений Пенлеве? В частности, можно ли использовать эту же процедуру для вывода $q$-дискретных уравнений Пенлеве? Заметим, что до настояшего времени только дискретные уравнения Пенлеве разностного типа были выведены с помощью этой процедуры.

4. В работах Окамото [14-17] показано,что уравнения Пенлеве PII-PVI допускают симметрии под действием афффинной группы Вейля $A_{1}^{(1)}$, которые связаны с присоединеными преобразованиями Беклунда. Связь между симметрией аффинной групшы Вейля и структурой рациональных решений PII-PVI изучена в работах [8, 69, 75-79]. Связь меж ду аффинной группой Вейля, уравнениями Пенлеве и дискретными уравнениями обсуждалась недавно в статье Ноуми и Ямады [80], хотя в ней эти дискретные уравнения и не были отнесены к дискретным уравнениям Пенлеве. Это, очевидно, требует дальнейшего исследования.

5. Может ли эта процедура быть применена для получения конечных периодических разностных уравнений, т.е. разностных уравнений, в которых $x_{n+N}=x_{n}$ для всех $n$ при фиксированном $N$ ?

6. Можно ли распространить эту процедуру на уравнения высших порядков, например на иерархию РII (ср. [81])?

7. Что получится, если применить эту процедуру к преобразованиям Беклунда для дискретных уравнений Пенлеве?

Благодарности. Мы хотим поблагодарить Налини Джоши за полезные замечания и многое объясняюшее обсуждение результатов. П. А. Кларксон и Е. Л. Менсфилд благодарны Отделению теоретической математики, Университет Аделаиды, Австралия, за гостеприимство во время их визита, когда было начато это исследование, частично поддержанное Австралийским исследовательским советом. П. А. Кларксон также благодарит Институт математических научных исследований, Беркли, США (MSRI), за гостеприимство при завершении этой статьи. Исследования в MSRI были частично поддержаны NSF, грант DMS-9701755. Работа X. Н. Вебстер была поддержана Институтом математики и статистики (аспирантский грант Кентского университета). 


\section{Список литературы}

[1] E. Picard. Compt. Rend. Acad. Sci. Paris. 1887. V. 104. P. 41-43.

[2] Э. Л. Айнс. Обыкновенные дифференциальные уравнения. Харьков: ГНТИ Украины, 1939.

[3] M. J. Ablowitz, P. A. Clarkson. Solitons, nonlinear evolution equations and inverse scattering. L.M.S. Lect. Notes Math. V. 149. Cambridge: C.U.P., 1991.

[4] H. Airault. Stud. Appl. Math. 1979. V. 61. P. 31-53.

[5] A. P. Bassom, P. A. Clarkson, A. C. Hicks. Stud. Appl. Math. 1995. V. 95. P. 1-71.

[6] В. И. Громак. Диф. уравн. 1978. Т. 14. С. 2131-2135.

[7] K. Kajiwara, T. Masuda. A generalization of determinant formulas for the solutions of Painlevé II and XXIV equations. solv-int/9903014.

[8] K. Kajiwara, T. Masuda. On the Umemura polynomials for the Painleve III equation. solv-int/9903015.

[9] K. Kajiwara, Y. Ohta. J. Math. Phys. 1996. V. 37. P. 4393-4704.

[10] K. Kajiwara, Y. Ohta. J. Phys. A. 1998. V. 31. P. 2431-2446.

[11] A. E. Milne, P. A. Clarkson, A. P. Bassom. Stud. Appl. Math. 1997. V. 98. P. 139-194.

[12] Y. Murata. Funkc. Ekvacioj. Ser. Int. 1985. V. 28. P. 1-32.

[13] Y. Murata. Nagoya Math. J. 1995. V. 139. P. 37-65.

[14] K. Okamoto. Ann. Mat. Pure Appl. 1987. V. 146. P. 337-381.

[15] K. Okamoto. Japan. J. Math. 1987. V. 13. P. 47-76.

[16] K. Okamoto. Math. Annal. 1986. V. 275. P. 221-255.

[17] K. Okamoto. Funkc. Ekvacioj. Ser. Int. 1987. V. 30. P. 305-332.

[18] A.S. Fokas, M. J. Ablowitz. J. Math. Phys. 1982. V. 23. P. 2033-2042.

[19] A.S. Fokas, U. Mugan, M. J. Ablowitz. Physica D. 1988. V. 30. P. 247-283.

[20] В. И. Громак. Диф. уравн. 1975. Т. 11. С. 373-376.

[21] U. Mugan, A.S. Fokas. J. Math. Phys. 1992. V. 33. P. 2031-2045.

[22] H. Flaschka, A. C. Newell. Commun. Math. Phys. 1980. V. 76. P. 65-116.

[23] A.R. Its, V. Yu. Novokshenov. The Isomonodromic Deformation Method in the Theory of Painlevé Equations. Lect. Notes in Math. V. 1191. Berlin: Springer-Verlag, 1986.

[24] K. Okamoto. J. Fac. Sci. Univ. Tokyo, Sect. IA Math. 1986. V. 33. P. 575-618.

[25] A. P. Magnus. J. Comp. Appl. Anal. 1995. V. 57. P. 215-237.

[26] E. Brézin, V. A. Kazakov. Phys. Lett. B. 1990. V. 236. P. 144-150.

[27] D. J. Gross, A. A. Migdal. Phys. Rev. Lett. 1990. V. 64. P. 127-130.

[28] V. Periwal, D. Shevitz. Phys. Rev. Lett. 1990. V. 64. P. 1326-1329.

[29] B. Grammaticos, A. Ramani, V. Papageorgiou. Phys. Rev. Lett. 1991. V. 67. P. 1825-1828.

[30] A. Ramani, B. Grammaticos, J. Hietarinta. Phys. Rev. Lett. 1991. V. 67. P. 1829-1832.

[31] J. Hietarinta, C. Viallet. Phys. Rev. Lett. 1998. V. 81. P. 325-328.

[32] M. Jimbo, H. Sakai. Lett. Math. Phys. 1996. V. 38. P. 145-154.

[33] M. Jimbo, H. Sakai, A. Ramani, B. Grammaticos. Phys. Lett. A. 1996. V. 217. P. 111-118.

[34] B. Grammaticos, A. Ramani. The hunting for the discrete Painlevé VI is over. solv-int/9901006.

[35] A. P. Bassom, P. A. Clarkson. Phys. Lett. A. 1994. V. 194. P. 358-370.

[36] P. A. Clarkson, H. N. Webster. Chaos, Solitons and Fractals (в печати).

[37] C. Cresswell, N. Joshi. J. Phys. A. 1999. V. 32. P. 655-669.

[38] B. Grammaticos, F. W. Nijhoff, V. Papageorgiou, A. Ramani, J. Satsuma. Phys. Lett. A. 1994. V. 185. P. 446-452.

[39] B. Grammaticos, F.W. Nijhoff, A. Ramani. Discrete Painlevé equations. In: The Painlevé Property, One Century Later. CRM series in Mathematical Physics. Ed. R. Conte. New York: Springer-Verlag, 1999. P. 413-516.

[40] J. Hietarinta, K. Kajiwara. Rational solutions to d-PIV. In: Symmetries and Integrability of Difference Equations. LMS Lect. Notes Series. V. 255. Eds. P. A. Clarkson, F. W. Nijhoff. Cambridge: C.U.P., 1999. P. 206-216.

[41] N. Joshi, A. Ramani, B. Grammaticos. Phys. Lett. A. 1998. V. 249. P. 59-62.

[42] K. Kajiwara. The discrete Painlevé II equation and the classical special functions. In: Symmetries and Integrability of Difference Equations. LMS Lect. Notes Series, V. 255. Eds. P. A. Clarkson, F. W. Nijhoff. Cambridge: C.U.P., 1999. P. 217-227.

[43] K. Kajiwara, Y. Ohta, J. Satsuma. J. Math. Phys. 1995. V. 36. P. 4162-4174. 
[44] K. Kajiwara, Y. Ohta, J. Satsuma, B. Grammaticos, A. Ramani. J. Phys. A. 1994. V. 27. P. 915-922.

[45] K. Kajiwara, K. Yamamoto, Y. Ohta. Phys. Lett. A. 1997. V. 232. P. 189-199.

[46] F. W. Nijhoff, J. Satsuma, K. Kajiwara, B. Grammaticos, A. Ramani. Inverse Problems. 1996. V. 12. P. $697-716$.

[47] Y. Ohta, K. Kajiwara, J. Satsuma. Bilinear structure and exact solutions of the discrete Painlevé I equation. In: Symmetries and Integrability of Difference Equations. CRM Proc. Lecture Notes. V. 9. Eds. P. Winternitz, D. Levi. Providence, RI: Amer. Math. Soc., 1996. P. 265-268.

[48] Y. Ohta, A. Ramani, B. Grammaticos, K.M.Tamizhmani. Phys. Lett. A. 1996. V. 216. P. 255-261.

[49] A. Ramani, B. Grammaticos. Physica A. 1996. V. 228. P. 160-171.

[50] A. Ramani, B. Grammaticos, J. Satsuma. J. Phys. A. 1995. V. 28. P. 4655-4665.

[51] A. Ramani, Y. Ohta, J. Satsuma, B. Grammaticos. Commun. Math. Phys. 1998. V. 192. P. $67-76$.

[52] J. Satsuma, K. Kajiwara, B. Grammaticos, J. Hietarinta, A. Ramani. J. Phys. A. 1995. V. 28. P. $3541-3548$.

[53] K. M. Tamizhmani, B. Grammaticos, A. Ramani. Lett. Math. Phys. 1993. V. 29. P. 49-54.

[54] K. M. Tamizhmani, A. Ramani, B. Grammaticos, K.Kajiwara. J. Phys. A. 1998. V. 31. P. $5799-5810$.

[55] K. M. Tamizhmani, A. Ramani, B. Grammaticos, Y. Ohta. Lett. Math. Phys. 1996. V. 38. P. 289-296.

[56] A.S. Fokas, B. Grammaticos, A. Ramani. J. Math. Anal. Appl. 1993. V. 180. P. 342-360.

[57] B. Grammaticos, A. Ramani. Discrete Painlevé equations: derivation and properties. In: Application of Analytic and Geometric Methods to Nonlinear Differential Equations. NATO ASI Series C. V. 413. Ed. P. A. Clarkson. Dordrecht: Kluwer, 1993. P. 299-313.

[58] B. Grammaticos, A. Ramani. J. Phys. A. 1998. V. 31. P. 5787-5798.

[59] В. И. Громак, В. В. Цегельник. Диф. уравн. 1994. Т. 30. С. 1118-1124.

[60] В. И. Громак, В. В. Цегельник. Диф. уравн. 1996. Т. 32. С. 1018-1023.

[61] В. В. Цегельник. ТМФ. 1995. Т. 102. С. 364-366.

[62] В. В. Цегельник.Диф. уравн. 1996. Т. 32. С. 1434-1435.

[63] Э. Уиттекер, Джс. Ватсон. Курс современного анализа. М.: Физматгиз, 1962.

[64] Н. А. Лукашевич. Диф. уравн. 1971. Т. 7. С. 1124-1125.

[65] Н. А. Лукашевич. Диф. уравн. 1967. Т. 3. С. 771-780.

[66] A.В. Китаев.. 1991 (частное сообщение).

[67] A.S. Fokas, A.R. Its, A. V. Kitaev. Commun. Math. Phys. 1991. V. 142. P. 313-344.

[68] A. P. Bassom, P. A. Clarkson, A. C. Hicks. Adv. Diff. Eqns. 1995. V. 1. P. 175-198.

[69] H. Umemura, H. Watanabe. Nagoya Math. J. 1997. V. 148. P. 151-198.

[70] P. A. Clarkson. Europ. J. Appl. Math. 1990. V. 1. P. 279-300.

[71] V. I. Gromak. Bäcklund transformations of Painlevé equations and their applications. In: The Painlevé Property, One Century Later. CRM series in Mathematical Physics. Ed. R. Conte. New York: Springer-Verlag, 1999. P. 687-734.

[72] Н. А. Лукашевич. Диф. уравн. 1965. Т. 1. С. 731-735.

[73] Н. А. Лукашевич. Диф. уравн. 1967. Т. 3. С. 1913-1923.

[74] E. L. Mansfield, H. N. Webster. Stud. Appl. Math. 1998. V. 101. P. 321-341.

[75] M. Noumi, S. Okada, K. Okamoto, H. Umemura. Special polynomials associated with the Painlevé equation II. In: Integrable Systems and Algebraic Geometry. Eds. M.-H. Saito, Y. Shimizu, R. Ueno. Singapore: World Scientific, 1998. P. 349-372.

[76] H. Umemura. Special polynomials associated with the Painlevé equations I. Preprint, 1996.

[77] H. Umemura, H. Watanabe. Nagoya Math. J. 1998. V. 151. P. 1-24.

[78] M. Noumi, Y. Yamada. Commun. Math. Phys. 1998. V. 199. P. 281-295.

[79] M. Noumi, Y. Yamada. Phys. Lett. A. 1998. V. 247. P. 65-69.

[80] M. Noumi, Y. Yamada. Nagoya Math. J. (в печати); Symmetries in the fourth Painlevé equation and Okamoto polynomials. q-alg/9708018.

[81] P. A. Clarkson, N. Joshi, A. Pickering. Inverse Problems. 1999. V. 15. P. 175-187. 